\title{
VIRTUAL MIMO RADAR USING OFDM-CDM WAVEFORMS
}

\author{
S. Zazo, B. Béjar, J. Grajal
}

Dpto: Señales, Sistemas y Radiocomunicaciones-ETSIT. Universidad Politécnica de Madrid phone: + (34) 915495700 (Ext: 4042), fax: + (int. 34) 913367350, email: santiago@gaps.ssr.upm.es

\begin{abstract}
This paper addresses a new perspective on the exploitation of diversity resembling recent seminal proposals with multiple antennas known as MIMO radar. Our focus pursues similar advantages as spatial MIMO systems but intending to achieve the desired resistance over fading or/and SNR increase without relying on multiple antennas. We design an OFDM-CDM waveform well inspired in modern communications systems that creates a virtual MIMO system operating on the artificial $2 D$ domain formed by a set of well separated carriers (OFDM) and several OFDM symbols each one modulated by orthogonal codes (CDM). We consider the most general scenario with moving targets and large size targets originating an equivalent time variant and frequency selective channel model. Our proposal proceeds in two steps, a first one to reorthogonalize the transmitted set of OFDM signal by proper time and frequency synchronization (this stage provides range and velocity estimators), and a second one based on Neyman Pearson detection improved by the diversity gain.
\end{abstract}

\section{MOTIVATION}

Application of spatial MIMO (Multiple Input - Multiple Output) principles has attracted large research interest in the communication field due to the great improvements predicted by recent theoretical results [Te199, Fos98]. Presently all important applications from mobile to WLANs, xDSL or sensor networks and working towards practical implementations to increase their performances exploiting diversity at reception by optimum combining, space time coding to emulate the diversity gain at transmitter side, beamforming gain and even also spatial multiplexing [Pau03]. Therefore, it was just a question of time that these advantages may be applied to the radar field. Several recent papers are revisiting the classical radar objectives applying these concepts claiming much higher resolution capabilities and improved detection probabilities [Fis04, Fis06, Leh07]. Although these contributions are clearly opening a new paradigm in the radar field [Lev04], we would like to emphasize two key issues: on one hand, deployment of several multi site antennas may not be applicable in many scenarios and any case the cost increase may be unaffordable. On the other, the statistical MIMO (SMIMO in the sequel) radar is based on the assumption of orthogonality among the received waveforms in order to create many independent radars. This last hypothesis is probably limited to very simple cases and in practice correlation among received waveforms will decrease performance.
From our point of view, we propose a different means to achieve the same appealing features of spatial MIMO systems using just one antenna (single site or multisite) per link inducing diversity and array gain exploiting the OFDMCDM characteristics. This architecture is robust to work in moving and large size target scenarios using the well known capability of OFDM systems to deal with multipath channels. Doppler robustness is compensated by frequency estimation in an equivalent way as offsets are reduced in standard communications.

\section{SIGNAL MODEL}

The received signal in the S-MIMO radar is based on a simple model as described by equation (1)

$$
\mathbf{r}_{\text {S-MIMO }}(t)=\sqrt{P} \mathbf{H}_{T} \mathbf{s}(t-\tau)+\mathbf{n}(t)
$$

where $\mathbf{n}(t)$ is a AWGN noise signal with $\sigma^{2}$ power, $P$ is the transmitted power (assumed 1 in the sequel), $\mathbf{H}_{T}(M \times N)$ is the instantaneous mixture among the set of transmitted waveforms $\mathbf{s}(t)$ from every transmitting antenna to every receiving antenna (subscript $T$ means that we are talking of MIMO systems in the time domain. In OFDM, it is more convenient to express the process in the frequency domain where we will drop the subscript for clarity reasons). Statistics of matrix $\mathbf{H}_{T}$ may range from fully correlated (related to close located antennas and beamforming principles) to uncorrelated typically related to sparse configurations. While the former provides SNR gain by the coherence of the array factor, that is range increase, the latter provides diversity gain meaning robustness against target scintillations.

Equation (1) shows two important simplifications that are clearly conditioning the large improvement of S-MIMO radar in front of standard approaches. Let us show a more general model in equation (2) including Doppler effect $\left(f_{D}\right)$ and multipath $L$-size (larger size reflectors)

$$
\mathbf{r}_{G}(t)=\sqrt{P} e^{j 2 \pi \delta_{D^{l}}} \sum_{k=1}^{L} \mathbf{H}_{T k} \mathbf{s}\left(t-\tau_{k}\right)+\mathbf{n}(t)
$$

Performance of S-MIMO is based on simple receivers per antennas as a bank of matched filters to the transmitted waveforms. As they are supposedly orthogonal, you have $M N$ independent radars. This orthogonality preservation applies if channel model follows equation (1) but clearly fails in the more general case. To the best of our knowledge, there is no evaluation of the S-MIMO performance degradation if equation (2) applies.

Our proposal follows the scenario described by equation (2) for the SISO case. Multipath distortion is eliminated by the 
cyclic prefix of the OFDM symbol while velocity is estimated designing a specific OFDM feature for this purpose. After correction we retrieve an equivalent MIMO system with orthogonality preservation.

As mentioned above, we will use a waveform based on $K$ consecutive OFDM symbols each one with $2 N$ carriers separated $\Delta_{f}$ : We will assume that this separation is enough to guarantee uncorrelated fading characteristic. Every arbitrary OFDM symbol has a specific structure where only alternate carriers are active. Active carriers in every OFDM symbol are modulated by one different code belonging to any set of orthogonal functions as Walsh-Hadamard. The reasoning behind this structure is double: modulation of alternate carriers creates time duplicity of the symbol in two identical halves that may be used for robust time (range) and frequency offset (Doppler) estimation following [Sch97]. On the other hand, a certain number of $K \leq N$ consecutive symbols are transmitted in order to increase the autocorrelation properties of the transmitted signal and also minimize possible interference coming from close targets that may overlap the echoes. This number $K$ large will provide improved detection and estimation features but also will reduce the target discrimination capabilities. In practice, there should be a trade-off depending on the specific scenario. In the sequel, we will consider these figures as parameters. This system may operate as a pulsed radar or continuous wave depending on the scenario's target. We will emphasize the pulsed case because it is simpler to explain but it may be extended to the other option with minimum effort. In the frequency domain, the set of transmitted symbols may be represented in a timefrequency matrix $\mathbf{S}$ :

$$
\mathbf{S}=\sqrt{2}\left[\begin{array}{llll}
\tilde{\mathbf{c}}_{1} & \tilde{\mathbf{c}}_{2} & \cdots & \tilde{\mathbf{c}}_{K}
\end{array}\right]
$$

Where, as mentioned, $\widetilde{\mathbf{c}}_{k}$ represents a $2 N$ vector with alternate zeroes and values according to the $k$ Walsh Hadamard code. In the discrete time domain transmitted symbols are represented as the concatenation of the $2 N$ IDFT $\left(\mathbf{F}^{-1}\right.$ as a matrix operator) of the codes and $\mathbf{M}$ is the linear transformation to add the cyclic prefix $((2 N+L) \times 2 N)$ :

$$
\mathbf{s}=\sqrt{2}\left[\begin{array}{c}
\mathbf{M F}^{-1} \tilde{\mathbf{c}}_{1} \\
\mathbf{M F}^{-1} \tilde{\mathbf{c}}_{2} \\
\vdots \\
\mathbf{M F}^{-1} \tilde{\mathbf{c}}_{K}
\end{array}\right]
$$

Denoting $\mathbf{h}_{T}$ as the impulse response of the incoming echoes, $\mathbf{H}_{T}=$ Toe $\left(\mathbf{h}_{T}\right)$ is the Toeplitz matrix representation of the linear convolution with $\mathbf{h}_{T}$, and $\mathbf{W}$ is a diagonal matrix whose elements are the increasing phase shift due to the Doppler frequency normalized at the sampling rate, received signal becomes:

$$
\mathbf{x}=\mathbf{W H}_{T} \mathbf{s}+\mathbf{n}
$$

Figure 1 shows the block diagram of the receiver where clearly we want to remark two stages: a first one devoted to synchronize the received sequence in time and frequency that plays the role of range and velocity estimators. These parameters allow the receiver signal to be reorthogonalized in order to make possible the diversity achievement and/or SNR increase. Second step performs standard detection using
Neyman-Pearson criteria that may work under two hypotheses: on one hand, if channel estimator is possible, coherent processing applies with the consequent improvement. If not, detection based on the vector norm may also be applied. From the perspective of communications, channel estimation in OFDM is very simple because after proper synchronization and suitable choice of the cyclic prefix length longer than the maximum expected delay, flat fading applies per carrier. Therefore we would like to emphasize that with minimum extra complexity, this architecture provides coherent detection.

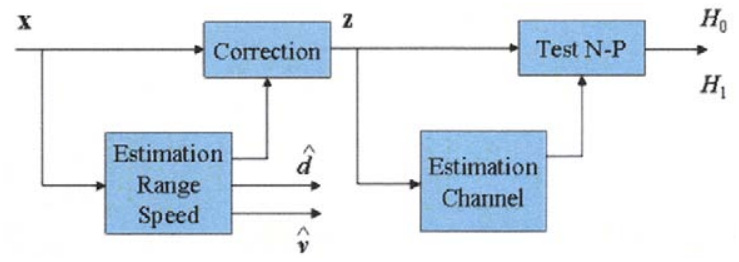

Fig. 1: Block diagram of our system.

\section{OUR PROPOSAL. ESTIMATION STAGE}

\subsection{Range and speed estimation}

Range and speed estimation is essentially the same problematic faced by an OFDM modem when it intends to synchronize in time and frequency. There are several methods to cope with this task but one of the most robust schemes are based on the special time duplicated structure of the training symbol proposed in [Sch97]. As mentioned before, transmission over alternate carriers creates the time duplication structure. The procedure is quite simple working in two steps: first step is just two consecutive sliding windows size $N$, correlating both identical halves to perform a time metric defined as (for just one symbol).

$$
\begin{gathered}
M(d)=\frac{|P(d)|^{2}}{(R(d))^{2}} \\
P(d)=\sum_{m=1}^{N-1} x_{d+m}^{*} x_{d+m+N} \quad R(d)=\sum_{m=0}^{N-1}\left|x_{d+m+N}\right|^{2}
\end{gathered}
$$

Equation (6) may be easily generalized to match $K$ consecutive OFDM symbols achieving the corresponding improvements due to the noise decorrelation. Clearly,

$$
\hat{d}_{o p t}=\max _{d} M(d)
$$

Once range has been estimated, the carrier frequency offset (and so target's speed) is obtained from the phase estimation

$$
\hat{\phi}=\operatorname{angle}\left(P\left(\hat{d}_{\text {opt }}\right)\right)
$$

However, it is important to remark the ambiguity of this procedure where maximum Doppler frequency is limited to the intercarrier separation. So, reduced ambiguity means increasing bandwidth or reducing number of carriers that also penalizes in SNR and diversity gain.

3.2 Signal model after time and frequency acquisition After proper correction in time and frequency, OFDM symbols are orthogonal providing a simple representation in the frequency domain for the $k$-OFDM symbol:

$$
\mathbf{z}_{k}=\mathbf{H}_{k} \mathbf{c}_{k}+\mathbf{n}_{k}
$$


Where $\mathbf{H}_{k}$ is a diagonal matrix whose entries are the complex attenuation at the corresponding frequencies and equivalently the target's response for each frequency. In the sequel we will assume that carriers are separated enough in order to claim for them as uncorrelated. $c_{k}$ are the scaled $\sqrt{2}$ WalshHadamard codes when zeroes are eliminated. Stacking the $K$ symbols in one vector we have

$$
\mathbf{z}=\left[\begin{array}{c}
\mathbf{z}_{q} \\
\mathbf{z}_{2} \\
\vdots \\
\mathbf{z}_{K}
\end{array}\right]=\left[\begin{array}{cccc}
\mathbf{H}_{1} & 0 & 0 & 0 \\
0 & \mathbf{H}_{2} & 0 & 0 \\
0 & 0 & \ddots & 0 \\
0 & 0 & 0 & \mathbf{H}_{K}
\end{array}\right]\left[\begin{array}{c}
\mathbf{c}_{q} \\
\mathbf{c}_{2} \\
\vdots \\
\mathbf{c}_{K}
\end{array}\right]=\tilde{\mathbf{H}} \mathbf{c}+\mathbf{n}
$$

As mentioned before, we may assume that we are able to estimate $\mathbf{H}_{k}$ in a very straightforward way.

\section{DETECTION STAGE}

For the detection stage we will assume to extreme models for the time correlation among the frequency responses: on one hand, fully correlated modeling a Swerling 1 target and on the other, fully uncorrelated to represent the Swerling 2 case. In the first case, the target's response is stable along the $K$ symbols and diversity of order $N$ is simply due to the uncorrelated frequency response but also there is a SNR increase due to averaging over $K$ OFDM symbols. This approach resembles, from the detection point of view, the S-MIMO concept where $N$ antennas at transmitter side are well separated while $K$ antennas are being used at receiver for beamfoming purposes with $K$ SNR improvement. In the latter, there is no SNR increase due to noise averaging but we are able to achieve a diversity order $N K$ due to the uncorrelated frequency response per symbol and also the uncorrelated evolution in time along the $K$ OFDM symbols. This is identical to the S-MIMO scenario where $N$ sparse transmitting antennas and $K$ well separated receiving antennas are considered creating $N K$ independent radars.

Defining $H_{0}$ as the hypothesis that target does not exist and $H_{1}$ that it exist, we are going to consider two options for processing incoming data: coherent (channel estimation is activated) and non coherent. Test criteria are the following

$$
\begin{array}{ll}
\text { Coherent : } & T_{C}=\Re\left\{\mathbf{c}^{H} \tilde{\mathbf{H}}^{H} \mathbf{z}\right\}_{<}^{>} \delta \\
\text { Non coherent } & T_{N C}=\|w(\mathbf{z})\|_{<}^{2} \delta
\end{array}
$$

Where thresholds will be defined following the Neyman Person criteria for a certain fixed probability of false alarm $\left(P_{F A}\right)$. $w(\mathbf{z})$ means a simple preprocessing to perform in the correlated non coherent case to average noise.

\subsection{Coherent processing}

This case is identical for the full correlated and uncorrelated cases because $\tilde{\mathbf{H}}$ is known (in practice there should be some difference because in the second case channel estimation variance will be larger, but we are not going to consider this aspect in this paper). Defining

$$
m=\mathbf{c}^{H} \tilde{\mathbf{H}}^{H} \mathbf{H e}
$$

$$
\left\{\begin{array}{llc}
H_{0} & T_{C}=\Re\left\{\mathbf{c}^{H} \tilde{\mathbf{H}}^{H} \mathbf{n}\right\} & N\left(0, \sigma^{2}\right) \\
H_{1} & T_{C}=m+\Re\left\{\mathbf{c}^{H} \tilde{\mathbf{H}}^{H} \mathbf{n}\right\} & N\left(m, \sigma^{2}\right)
\end{array}\right.
$$

This simple test follows the criteria [Bar05]

$$
T_{C}>\frac{\sigma^{2}}{2 m} \ln \eta+\frac{m}{2}=\gamma
$$

The corresponding probability of detection $P_{D}$ and probability of false alarm $P_{F A}$ are well known

$$
\begin{aligned}
& P_{F A}=Q\left(\frac{\gamma}{\sigma}\right) \\
& P_{D}=Q\left(\frac{\gamma-m}{\sigma}\right)
\end{aligned}
$$

Applying the Neyman-Person strategy, for a certain $P_{F A}$ we get a $P_{D}$ given by,

$$
P_{D}=Q\left(Q^{-1}\left(P_{F A}\right)-\frac{m}{\sigma}\right)
$$

In practice, assuming normalized $\tilde{\mathbf{H}}, m=2 \sqrt{N K}$ relying on the ergodicity concept where time averaging approximates statistical mean.

\subsection{Non coherent processing}

This case shows a different behavior for the correlated and uncorrelated scenarios. If $\mathbf{H}$ is assumed Gaussian, received signal is averaged over time in the full correlated case to reduce noise variance but also reducing the vector dimension while in the uncorrelated case there is no preprocessing before the test application. Let us define $\alpha$ as this Gaussian vector with normalized variance,

$$
\text { Uncorrelated: } \quad w(\mathbf{z})=\mathbf{z}=\sqrt{2} \boldsymbol{\alpha}+\mathbf{n} \quad(N K x \mathbf{l})
$$

Correlated : $\quad w(\mathbf{z})=\sqrt{2 K} \boldsymbol{\alpha}+\mathbf{n}$

Clearly, we have [Fis06]

$$
\text { Uncorrelated }:\|w(\mathbf{z})\|^{2} \rightarrow \begin{cases}H_{0} & \frac{\sigma^{2}}{2} \chi_{2 \mathrm{NK}}^{2} \\ H_{1} & \left(1+\frac{\sigma^{2}}{2}\right) \chi_{2 \mathrm{NK}}^{2}\end{cases}
$$

Correlated $:\|w(\mathbf{z})\|^{2} \rightarrow \begin{cases}H_{0} & \frac{\sigma^{2}}{2} \chi_{2 \mathrm{~N}}^{2} \\ H_{1} & \left(K+\frac{\sigma^{2}}{2}\right) \chi_{2 \mathrm{~N}}^{2}\end{cases}$

In this case, $P_{F A}$ and $P_{D}$ are

$$
\begin{gathered}
\text { Uncorr.: }\left\{\begin{array}{l}
P_{F A}=P\left\{T>\delta \mid H_{0}\right\}=P\left\{\chi_{2 \mathrm{NK}}^{2}>\frac{2 \delta}{\sigma^{2}}\right\} \\
P_{D}=P\left\{T>\delta \mid H_{1}\right\}=P\left\{\chi_{2 \mathrm{NK}}^{2}>\frac{2 \delta}{\sigma^{2}+2}\right\}
\end{array}\right. \\
\text { Corr.: }\left\{\begin{array}{l}
P_{F A}=P\left\{T>\delta \mid H_{0}\right\}=P\left\{\chi_{2 \mathrm{~N}}^{2}>\frac{2 \delta}{\sigma^{2}}\right\} \\
P_{D}=P\left\{T>\delta \mid H_{1}\right\}=P\left\{\chi_{2 \mathrm{~N}}^{2}>\frac{2 \delta}{\sigma^{2}+2 K}\right\}
\end{array}\right.
\end{gathered}
$$

Threshold $\delta$ is obtained in a similar way, 


$$
\begin{aligned}
& \text { Uncorrelated }: \delta=\frac{\sigma^{2}}{2} F_{2 N K}^{-1}\left(1-P_{F A}\right) \\
& \text { Correlated }: \delta=\frac{\sigma^{2}}{2} F_{2 N}^{-1}\left(1-P_{F A}\right)
\end{aligned}
$$

And the probability of detection finally becomes:

$$
\begin{aligned}
& \text { Uncorr.: } P_{D}=1-F_{2 N K}\left(\frac{\sigma^{2}}{\sigma^{2}+2} F_{2 N K}^{-1}\left(1-P_{F A}\right)\right) \\
& \text { Corr.: } P_{D}=1-F_{2 N}\left(\frac{\sigma^{2}}{\sigma^{2}+2 K} F_{2 N}^{-1}\left(1-P_{F A}\right)\right)
\end{aligned}
$$

\section{SIMULATION RESULTS}

Through this section several simulations are presented to evaluate the proposed scheme illustrating the behaviour of the estimation (range and Doppler) and detection stages. Performance of the proposed scheme is given separately for the two stages assuming for the second one that ideal correction has been carried out. All experiments have been run using Monte Carlo simulations with a number of repetitions equal to 10000 .

\subsection{Estimation stage}

Simulations regarding the estimation procedure have been run using $N=8$ subcarriers for different values of the number of OFDM symbols $K$ used to generate the waveform. The standard deviation of the two estimators (range and Doppler frequency) are given depending on different values of the number of OFDM symbols $K$. Both estimators are unbiased and the final estimates of the range (delay) and Doppler (phase) are obtained by averaging over the $K$ transmitted OFDM symbols.

In Figure 2 and 3 are represented the logarithm of the standard deviation for the range and Doppler estimators, respectively. It can be observed that, when increasing the number of symbols $K$, the variance of the estimators is reduced due to the averaging process. Two different curves are depicted for different SNR values $(0$ and $15 \mathrm{~dB})$ in order to show the improvement when increasing SNR.

From Figures 2 and 3 it becomes clear that even for low SNR scenarios and small number of subcarriers $N=8$, the used estimators for the proposed scheme perform quite well and that only small deviations from the real value are expected.

It is important to remark that performance on the estimation stage will strongly depend on the codes used for generating the waveforms. More precisely, if we select codes with better correlation properties, both estimates (range and Doppler) may be improved.

Time (range) resolution is limited by the chip interval of the OFDM symbol [Lev04],

$$
\frac{1}{2 N \Delta_{f}}
$$

where $\Delta_{f}$ is the intercarrier separation. Increasing separation or number of carriers means increasing bandwidth, therefore improving resolution. Increasing $K$ means making longer the time duration and increasing signal energy, providing improved detection capabilities and reduced estimation vari- ance. Design of these parameters depends very much on the scenario. It is worth to remark that this range estimator in fact is performing as a coarse first detector that do not exploit diversity as the second one. Its role is to improve the received signal to extract the maximum diversity provided by the target. In the future we have in mind to interconnect both detectors to design a joint procedure.

\subsection{Detection stage}

For the detection stage, different curves showing the probability of detection have been generated. In Figure 4, probability of detection is represented as a function of the SNR for a given false alarm probability while in Figure 5, SNR is fix and the probability of detection is given as a function of the false alarm probability. In both figures three different situations are considered depending on the target statistics (correlated or uncorrelated) and the availability of channel coefficients. As it was expected, coherent detection (with channel information) clearly outperforms the other two cases. With the proposed scheme based on multicarrier waveforms, coherent detection can be accomplished with minimum extra complexity and therefore improving the whole detection procedure.

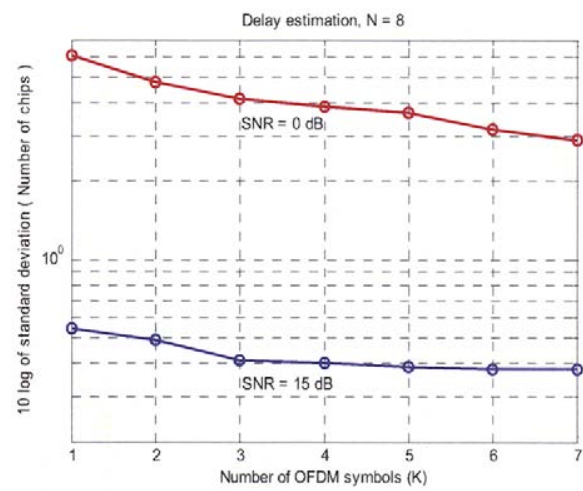

Fig. 2. Logarithm of the standard deviation of the range (delay) estimator for $N=8$ and different values of the SNR

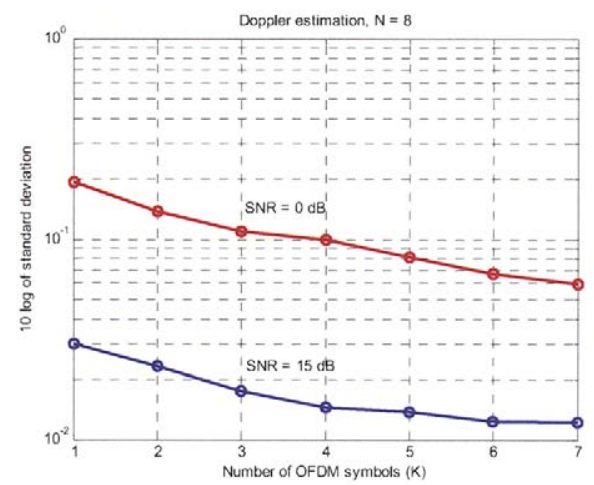

Fig. 3. Logarithm of the standard deviation of the Doppler estimator for $N=8$ and different values of SNR

Doppler estimator is really very robust as has been shown in the recent years widely being used as OFDM synchronizer. Its main drawback is the ambiguity related to the intercarrier space. Less ambiguity means extra bandwidth. However, 
using oversampling it is possible to reduce this ambiguity $N$ times without requiring more bandwidth [Zaz01].

The use of different codes per OFDM symbol intends to increase the robustness of the system in case that close targets cause waveforms overlapping. In principle, the target discrimination limit will be the OFDM symbol length $\left(1 / \Delta_{f}\right)$ because this is the minimum signal waveform from the first target that should be received with reduced interference from the second one in order to proceed with proper acquisition. Once this first one is detected, we propose to use interference cancellation schemes like those used in multiuser communications as BLAST [Fos03]. These ideas will be described in a forthcoming paper.

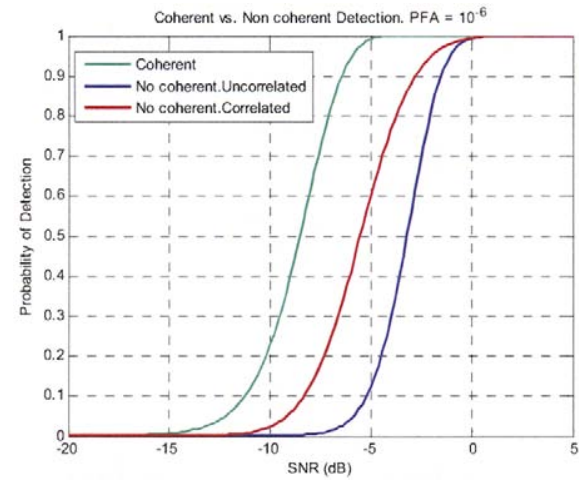

Fig. 4. Probability of detection as a function of the SNR for a given probability of false alarm equal to $10^{-6}$.

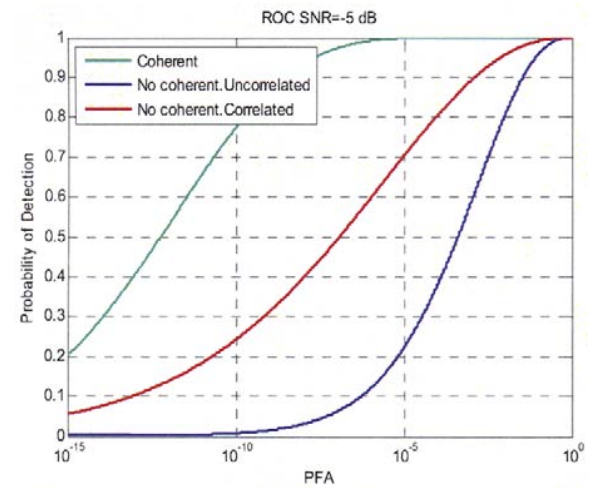

Fig. 5. Probability of detection as a function of the false alarm probability for a target $\mathrm{SNR}=-5 \mathrm{~dB}$.

The interplay between diversity and SNR increase is well known in communications: as observed in previous figures, diversity increases the slope of the probability curves while correlation shifts them. Depending on the scenario SNR, improvement due to one or other factor may be more valuable. In this case, at low SNR, correlated target provides more benefit.

\section{CONCLUSIONS}

This paper presents a new approach to the radar MIMO topic where a virtual multiple input multiple output system created with just one antenna using OFDM-CDM waveforms. This way, we are able to cope with more realistic scenarios including large size moving targets. Our proposal is based on a two steps procedure where range and velocity are estimated with improved resolution in order to restore the original orthogonality of OFDM waveforms and a second step where this diversity improves coherent or non coherent standard Neyman-Pearson detection. One of the key issues of this proposal is the single antenna motivation in order to reduce costs but clearly these ideas are perfectly compatible with having more antennas to increase diversity and more indeed become able to retrieve angular information. Eventually, it is important to remark that this approach may be interpreted as a detection procedure in two stages: a first one is the range detector that plays the role of 'activity detector' in order to decide if it is worth to extract the timefrequency characteristic of the signal due to the multipath and moving target. Second stage outperforms this first one extracting all the available diversity provided by the bandwidth (several well separated frequencies) and time duration.

\section{REFERENCES}

[Bar05] Barkat, M. Signal Detection and Estimation. Artech House Inc. 2005.

[Fis04] Fishler, E., Haimovich, A., Blum, R.S., Cimini, L.J., Chizhik, D., Valenzuela, R.A. MIMO Radar: An idea whose time has come. Proc. IEEE Conf. on Radar, April 2004

[Fis06] Fishler, E., Haimovich, A., Blum, R.S., Cimini, L.J., Chizhik, D., Valenzuela, R.A. Spatial Diversity in Radars. Models and Detection Performance. IEEE Transactions on Signal Processing, Vol. 54, NO.3, March 2006.

[Fos98] Foschini, G.J., Gans, M.J. On limits of Wireless Communications in a Fading Environment When Using Multiple Antennas. Wireless Personal Communications, Vol.6, NO.3, March 1998.

[Fos03] G.J. Foschini, D. Chizhik, M.J. Gans, C. Papadias, R.A. Valenzuela. Analysis and performance of some basic space-time architectures. IEEE Journal On Selected Areas In Communications, Vol. 21, NO 3, April 2003.

[Leh07]Lehmann, N.H., Fishler, E., Haimovich, A., Blum, R.S., Cimini, L.J., Chizhik, D., Valenzuela, R.A. Evaluation of Transmit Diversity in MIMO Radar Direction finding. IEEE Trans. on Signal Processing, Vol. 55, NO.5, May 2007. [Lev04] Levanon, N., Mozeson, E. Radar Signals. WileyIEEE Press, Julio 2004

[Pau03] Paulraj, A., Nabar, R., Gore, D. Introduction to Space-Time Wireless Communications. Cambridge University Press, 2003.

[Sch97] Schmidl, T.M., Cox, D.C. Robust Frequency and Timing Synchronization for OFDM. IEEE Transactions on Communications, Vol. 45, NO.12, December 1997.

[Tel99] I.E. Telatar. Capacity of multiantenna gaussian channels. European Transactions on Telecommunications, Vol. 10, November 1999.

[Zaz01] Zazo,S., Páez-Borrallo, J.M. Análisis of a New Frequency Synchronization Écheme in OFDM Systems. Signal Processing 81 (2001), pp. 1695-1704. 